\title{
Modulating perceptual complexity and load reveals degradation of the visual working memory network in ageing
}

\author{
Sobanawartiny Wijeakumar, ${ }^{\mathrm{a}, *}$, Vincent A. Magnotta ${ }^{\mathrm{b}}$, John P. Spencer ${ }^{\mathrm{a}}$ \\ a University of East Anglia, School of Psychology, Norwich NR4 7TJ, United Kingdom \\ b University of Iowa, Department of Radiology, Iowa City, IA 52242, USA
}

\begin{abstract}
A B S T R A C T
Previous neuroimaging studies have reported a posterior to anterior shift of activation in ageing (PASA). Here, we explore the nature of this shift by modulating load (1,2 or 3 items) and perceptual complexity in two variants of a visual working memory task (VWM): a 'simple' color and a 'complex' shape change detection task. Functional near-infrared spectroscopy (fNIRS) was used to record changes in activation in younger $(N=24)$ and older adults $(N=24)$. Older adults exhibited PASA by showing lesser activation in the posterior cortex and greater activation in the anterior cortex when compared to younger adults. Further, they showed reduced accuracy at loads 2 and 3 for the simple task and across all loads for the complex task. Activation in the posterior and anterior cortices was modulated differently for younger and older adults. In older adults, increasing load in the simple task was accompanied by decreasing activation in the posterior cortex and lack of modulation in the anterior cortex, suggesting the inability to encode and/or maintain representations without much aid from higher-order centres. In the complex task, older adults recruited verbal working memory areas in the posterior cortex, suggesting that they used adaptive strategies such as labelling the shape stimuli. This was accompanied by reduced activation in the anterior cortex reflecting the inability to exert top-down modulation to typical VWM areas in the posterior cortex to improve behavioral performance.
\end{abstract}

\section{Introduction}

Visual working memory (VWM) is a short term storage system for visual information that is critical for at least two central aspects of cognition - for the comparison of percepts that cannot be simultaneously foveated and for identifying changes in the world when they occur (Luck and Vogel, 1997). As a result, VWM is central to adaptive functions such as navigation, driving, reading, and so on. Healthy VWM processing is critical throughout the human life span, and deficits in VWM processing have serious consequences for performance during early development and late adulthood.

In healthy ageing, there are marked changes in this cognitive system. Decline in VWM is reported to start as early as in the fourth decade of life with a drop of 0.20 items per decade in both verbal and spatial working memory (Salthouse, 1994). Although these data suggest a dramatic decline in working memory function, other factors might also play a role: factors such as deconditioning of physiological processes following inactivity, arthritis, and declining motor abilities can affect behavioral measures such as accuracy and reaction times.
One way to move beyond motoric deficits is to investigate brain function alongside behavior in younger and older adults.

Studies of WM using functional magnetic resonance imaging (fMRI) implicate a distributed cortical network that spans the frontal, parietal, and temporo-occipital cortices (Druzgal and D'Esposito, 2003; Learmonth et al., 2002; Linden et al., 2003; Ma et al., 2014; Pessoa and Ungerleider, 2004; Postle, 2015; Rypma et al., 2002; Todd and Marois, 2005, 2004). In adults, there is generally an increase in blood oxygen level dependent (BOLD) activation as the number of items to be maintained in working memory (i.e., the load) is increased (Todd and Marois, 2004). In the ageing brain, this trend is maintained, but older adults tend to recruit larger areas of the prefrontal/frontal cortex and also show greater activation than younger adults when the demands of a task are low, for instance, with low working memory load (Cappell et al., 2010; Davis et al., 2008; Reuter-Lorenz and Campbell, 2008; Reuter-Lorenz et al., 2000). However, as load increases, older adults tend to show a drop or plateau in activation levels (Mattay et al., 2006). This has been referred to as the compensation-related utilization of neural circuits hypothesis (CRUNCH; Reuter-Lorenz and Campbell, 2008).

\footnotetext{
* Corresponding author.

E-mail address: S.Wijeakumar@uea.ac.uk (S. Wijeakumar).
} 
Some cognitive studies have also reported a posterior to anterior shift in ageing (PASA) wherein clusters in the ageing posterior cortex show reduced activation while clusters in the frontal cortex show increased activation relative to younger adults (Anderson, 2000; Cabeza et al., 2004; Davis et al., 2008; Dennis et al., 2007; Grossman et al., 2002; Gutchess, 2014; Reuter-Lorenz and Campbell, 2008; Reuter-Lorenz and Lustig, 2005; Reuter-Lorenz et al., 2000; Rypma and D'Esposito, 2000). More recent commentaries have discussed the nature of this shift in activation (see Monge and Madden (2016) for review). It is possible that deficits in perceptual processing in the posterior cortex lead to increased activation in higher-order processing centres in the anterior cortex. Alternatively, impoverished modulation from higher order centres in the anterior cortex might lead to problems in perceptual processing in the posterior cortex.

To further clarify the relationship between the posterior and anterior cortices in the ageing brain, the present study builds on a recent fMRI study that modulated perceptual complexity and load during a visual working memory task in young adults (Ambrose et al., 2016). In particular, this study used a 'simple' color change detection task and a 'complex' shape change detection task with varying loads. Results showed significantly lower WM capacities for the more complex task and a selective modulation of posterior cortex in response to this task manipulation. In particular, clusters in occipital cortex, ventral occipital cortex, intraparietal sulcus, superior intraparietal sulcus, and middle fusiform gyrus all showed an activation plateau at high WM loads for the simple task, but a precipitous decline in activation at the highest load for the complex task.

In the present study, we used the same modulation of task complexity in a VWM task with younger and older adults. The first central question was whether the older adults would show both lower VWM capacity overall, and also lower capacity for the more complex task relative to the simple task. If so, is the modulation of the brain in response to the task manipulation selective to posterior cortex such as with younger adults? This is a critical question with PASA. We know there is some form of posterior decline, but is there still the potential for selective task-specific posterior modulation? And does this modulation resemble the effect with younger adults with a precipitous decline in activation for the complex task? The alternative is that the modulation of the brain in response to task complexity happens primarily in the anterior brain. A central hypothesis in PASA is that the anterior brain might be compensating for posterior decline. If the activation of the anterior cortex is compensatory, we should see differences in this activation pattern as task complexity is manipulated. It is possible, of course, that both the posterior and anterior cortices are affected by the manipulation of task complexity. Here, the pattern of modulation should shed light on interactions between the posterior and anterior brain.

Most previous examinations of VWM at the neural level in adults have used fMRI. In the current study, we used functional near-infrared spectroscopy (fNIRS) because this technology is easier and more convenient to administer with older participants. fNIRS is an optical imaging technique where near-infrared light of two different wavelengths is shone through tissue and is selectively absorbed by oxy(HbO) and deoxy- (HbR) hemoglobin. fNIRS has been used to explore neural function underlying a wide range of sensory (Bortfeld et al., 2007; Chen et al., 2015; Plichta et al., 2011; Wijeakumar et al., 2012), motor (Gagnon et al., 2012; Huppert et al., 2006) and cognitive functions such as working memory, response inhibition, and taskswitching (Cutini et al., 2008; Rodrigo et al., 2014; Wijeakumar et al., 2017). fNIRS is a convenient technique for investigating brain function in response to VWM processing because the regions of interest (ROIs) are near the cortical surface. fNIRS is also cheaper than fMRI, making intensive study of age-related decline more feasible, and older adults do not have to remain completely still in a supine position for lengthy periods of time. Moreover, recent innovations in fNIRS analyses allow us to obtain voxel-wise measures of functional activation that can be directly compared to fMRI data (Wijeakumar et al., 2017, 2015).

\section{Materials and methods}

\section{Participants}

Twenty-eight younger and twenty-eight older participants took part in the experiment. All participants reported that they had normal or corrected-to-normal vision. When needed, corrected prescriptions were worn during the tasks. The final analyses consisted of twenty-four younger (age range: $25.4 \pm 4.3$ years old) and twenty-four older (age range: $70.5 \pm 5.2$ years old) participants. All participants signed an informed consent form approved by the Ethics Committee at the University of Iowa.

\section{Screening procedures}

Both groups of participants were asked to fill in questionnaires to assess if they had any health-related circumstances such as mental health problems, use of medication, or surgeries that would have an impact on task performance. All older participants completed the Montreal Cognitive Assessment to assess the presence of cognitive impairment. Participants who obtained a score of less than 26 (out of 30 points) were excluded. A score of less than 26 implied the presence of mild cognitive impairment. They also completed the Geriatric Depression Scale to identify the presence of depression. Those participants who obtained a score greater than 5 were excluded. A score of 5 or greater might indicate the presence of depression and the need for a more thorough clinical investigation.

\section{Stimuli and design}

Details of the experimental paradigms have been described elsewhere (Ambrose et al., 2016). Briefly, colors and shapes were used as stimuli. Colors were equally distributed in CIELAB 1976 color space. We used shapes based on Drucker and Aguirre's RFC-defined stimuli (Drucker and Aguirre, 2009). Four different colors and four different shapes were used. Each color or shape was separated by at least $90^{\circ}$ in feature space from the other colors or shapes. The experiments were created and run using E-prime version 2.0 on an HP computer.

Two variants of a Change Detection (CD) task were run (see Fig. 1). In the 'simple' color task, participants were presented with a memory array with colored stimuli of the same shape. After a brief delay, a test array was presented that was either the same as the memory array or one of the colors of the stimuli was changed. The shapes of the stimuli were held constant. In the 'complex' shape task, participants were presented with a memory array with stimuli of different shapes (all stimuli were of the same color). After a brief delay, a test array was presented that was either the same as the memory array or one of the stimuli had a different shape. In both tasks, participants had to indicate

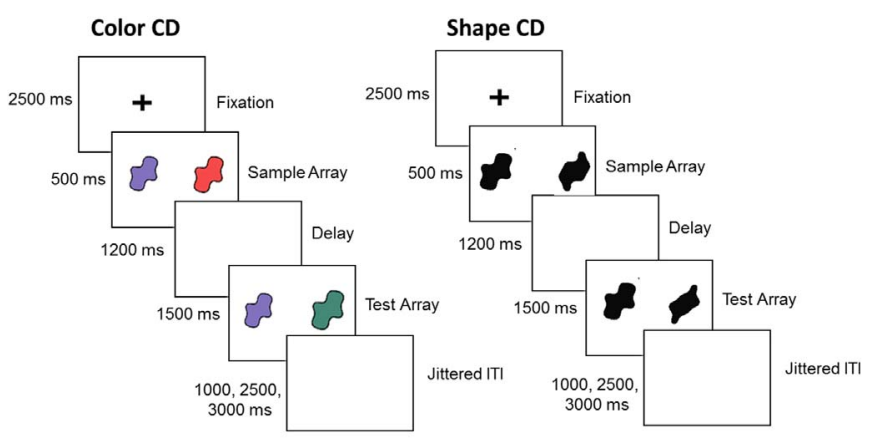

Fig. 1. 'Simple' color and 'complex' shape CD tasks. 
a.

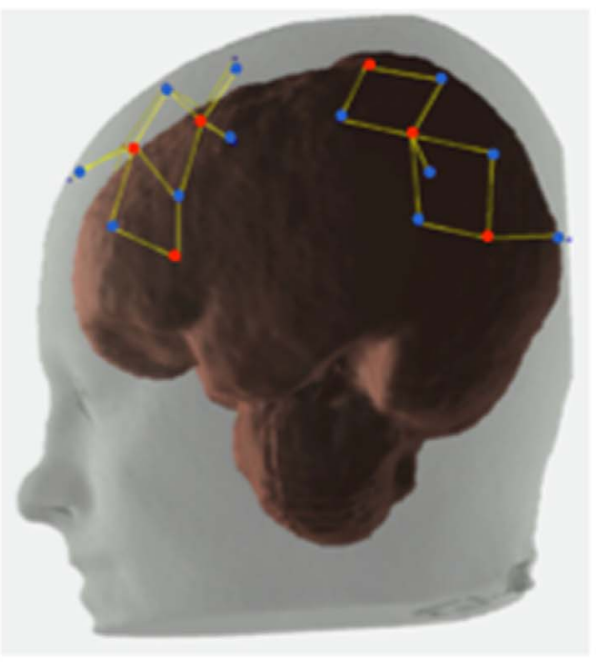

c.

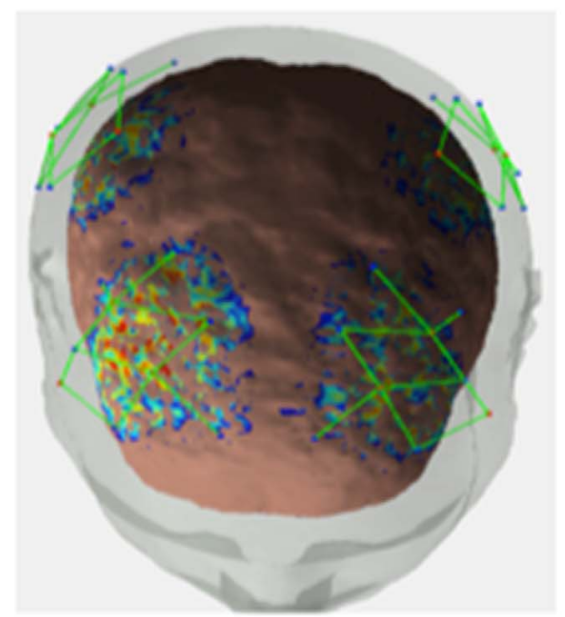

b.

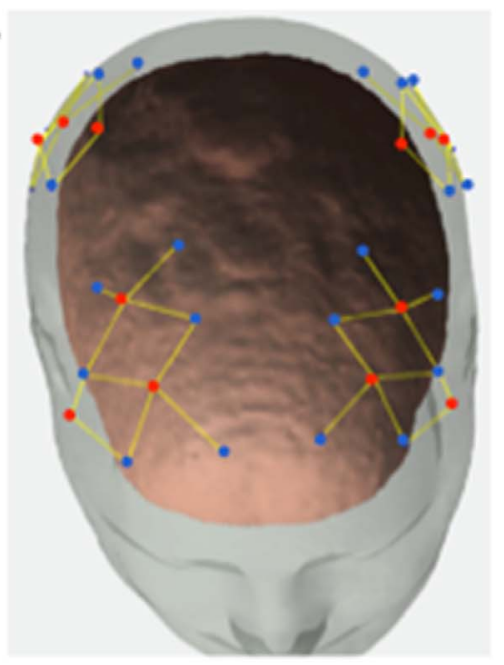

d.

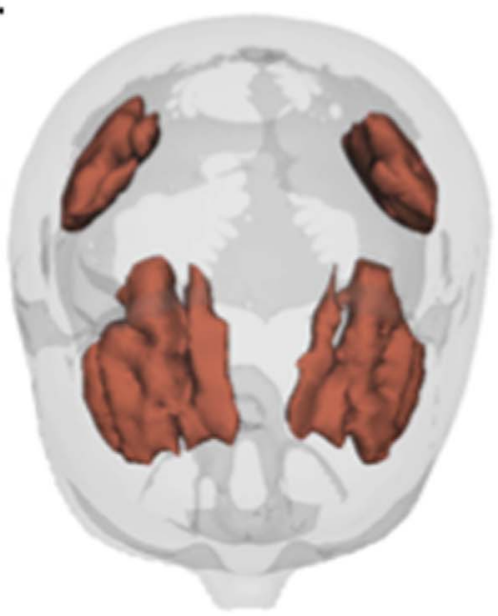

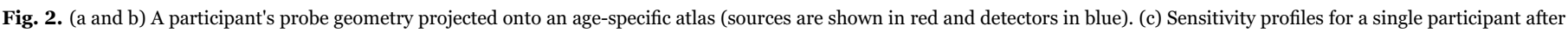

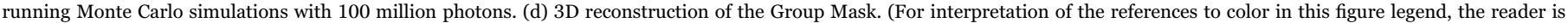
referred to the web version of this article.)

using a button whether the two arrays were the same or different. The participants were allowed to practice on a few trials to familiarize themselves with the tasks. The number of items presented (working memory load) varied between one, two or three items. Half of the participants performed the color task followed by the shape task, whilst the other half did the reverse.

Each trial began with a fixation cross that lasted for $2500 \mathrm{~ms}$. Then, a memory array was presented for $500 \mathrm{~ms}$ followed by a delay blank screen for $1200 \mathrm{~ms}$. Finally, this was followed by a test array for $1500 \mathrm{~ms}$. Inter-trial intervals were jittered at $1000 \mathrm{~ms}$ (50\%), $2500 \mathrm{~ms}$ (25\%) or $3500 \mathrm{~ms}$ (25\%). Participants completed one run each for the color and shape tasks. Each run consisted of 120 trials with 40 randomized trials (20 same, 20 different) at each of the three load conditions.

\section{NIRS recording and processing}

\section{Instrumentation}

A TechEn CW6 system (12 sources and 24 detectors) with wavelengths of $830 \mathrm{~nm}$ and $690 \mathrm{~nm}$ were used to collect fNIRS data at $25 \mathrm{~Hz}$. Fiber optic cables carried light from the machine to a customized cap designed to collect $\mathrm{HbO}$ and $\mathrm{HbR}$ concentration. The probe geometry consisted of 36 channels with $3 \mathrm{~cm}$ source-detector separations and 4 channels with a source-detector separation of $1 \mathrm{~cm}$.
In total, there were 40 channels covering the frontal and parietal cortices. In our previous work, we optimized this probe geometry such that it covered ROIs from the fMRI VWM literature and was anchored to the 10-20 system of electrode placement so that the geometry could be scaled to different head sizes (Wijeakumar et al., 2015).

\section{Pre-processing fNIRS channel data}

fNIRS data were pre-processed using HOMER2 (www.nmr.mgh.harvard.edu/PMI/resources/homer2). Raw data were pruned to include signals between $80 \mathrm{~dB}$ and $130 \mathrm{~dB}$. Then, these signals were converted from intensity values to Optical Density (OD) units. Motion artifacts were detected using the motionArtifactByChannel function (tMotion $=1.0$, tMask $=1.0$, StdevThresh $=50$ and AmpThresh = 0.5) and the identified segments were corrected using the $t P C A$ function (Yücel et al., 2014) [tMotion = 1.0, tMask = 1.0, StdevThresh $=50$ and AmpThresh = 0.5], and finally, the StimRejection function was applied to reject any segments of data that remained uncorrected. The data were then band-pass filtered to include frequencies between 0.016 and $0.5 \mathrm{~Hz}$. OD data was then converted to concentration units using the modified Beer-Lambert law. General linear models (GLM) were run on the HbO and HbR data separately. Each GLM consisted of six regressors. Each regressor was created by convolving a modified gamma function with square wave duration of $12 \mathrm{~s}$ with the onset of each trial. The six regressors 
consisted of same and different trials for loads 1, 2 and 3. Regressors were also constructed from those channels that served as short sourcedetector pairs. Signals from the closest short source-detector pair were regressed from surrounding channels. A beta estimate was obtained for each condition, channel, chromophore and participant.

\section{Forward model}

We used atlases from the Neurodevelopmental MRI Database (Fillmore et al., 2015; Richards and Xie, 2015; Richards et al., 2016) for all participants. The age of each participant was used to choose the appropriate atlas from the database. Each atlas was segmented into separate volumes for gray matter, white matter, cerebro-spinal fluid, and scalp tissues and assigned different values using $3 \mathrm{dSeg}$ from AFNI (Analysis of Functional Neuroimaging). These volumes were converted into 3D surface meshes using HOMER2. We relied on the procedures used in Wijeakumar et al. (2017).

Scalp landmarks and probe geometry for each participant were digitized using a Polhemus Patriot Motion Sensor. These points were projected onto each participant's head atlas using a relaxation algorithm (AtlasViewerGUI - available within HOMER2: www.nmr.mgh. harvard.edu/PMI/resources/Homer2). Projection of the probe geometry onto a single participant's age-specific segmented atlas is shown in Fig. 2a and b. This probe geometry was used to run Monte Carlo simulations with 100 million photons to create sensitivity profiles for each channel (see Fig. 2c). The sensitivity profiles and head volumes were converted to NIFTI format. The sensitivity profiles for each participant were summed together and thresholded to include voxels with an optical density of greater than 0.0001 (see Wijeakumar et al., 2015). Each subject-specific mask was transformed to MNI space using 3dAllineate. All subject-specific masks were then summed together to create a single image. Only those voxels in this image that contained data from at least $75 \%$ of the participants were used to create the Group mask of size 18,224 voxels (see Fig. 2d)

\section{Image reconstruction}

We relied on methodology previously developed and validated with fMRI to combine the sensitivity profiles with channel-specific beta coefficients to generate voxel-wise estimates of $\mathrm{HbO}$ and $\mathrm{HbR}$ activation for each condition and participant (Wijeakumar et al., 2017). Briefly, the relationship between the hemodynamic response (beta coefficients from the channel-specific GLM described in Section 2.3.2) and delta optical density is given by:

$\beta_{d O D}^{\lambda}=p p f^{\lambda} \cdot d \cdot \varepsilon_{H b O}^{\lambda} \cdot \beta_{H b O}+p p f^{\lambda} \cdot d \cdot \varepsilon_{H b R}^{\lambda} \cdot \beta_{H b R}$

where, $d$ is the source-detector distance, $\varepsilon$ is the extinction coefficient for each wavelength $(\lambda)$ and $p p f$ is the partial pathlength factor.

After accommodating for the forward model and the channelspecific beta coefficients, Eq. (1) can be re-written as:

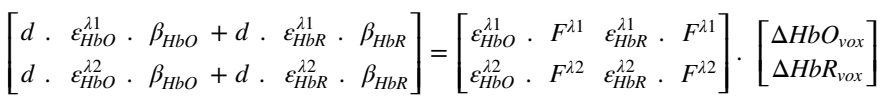

where, $F$ is the channel-wise sensitivity volumes from the Monte Carlo simulations. $\triangle H b O_{v o x}$ and $\Delta H b R_{\text {vox }}$ are voxel-wise relative changes in $\mathrm{HbO}$ and $\mathrm{HbR}$ concentrations - this is what we want to estimate in the image reconstruction process. We can re-write Eq. (2) as:

$Y=L . X$

where,

$\mathrm{Y}=\left[\begin{array}{l}\beta_{d O D}^{\lambda 1} \\ \beta_{d O D}^{\lambda 2}\end{array}\right]$

$\mathrm{L}=\left[\begin{array}{llll}\varepsilon_{H b O}^{\lambda 1} \cdot F^{\lambda 1} & \varepsilon_{H b R}^{\lambda 1} \cdot F^{\lambda 1} \\ \varepsilon_{H b R}^{\lambda 2} \cdot F^{\lambda 2} & \varepsilon_{H b R}^{\lambda 2} \cdot F^{\lambda 2}\end{array}\right]$ and $X=\left[\begin{array}{l}\Delta H b O_{v o x} \\ \Delta H b R_{v o x}\end{array}\right]$

To solve for $\mathrm{X}$, we used Tikhonov regularization and the system in Eq. (3) can be replaced by a 'regularized' system given by,

$X=\left(L^{T} L+\lambda \cdot I\right)^{-1} L^{T} . Y$

where $\lambda$ is a regularization parameter that determines the amount of regularization and $I$ is the identity operator. The solution to (4) can be found by minimizing the cost function (Calvetti et al., 2000),

cost $\min X=|L . X-Y|^{2}+\lambda .\left|X-X_{o}\right|^{2}$

where the size of the regularized solution is measured by the norm $\lambda$. | $X-\left.X_{0}\right|^{2} . \mathrm{X}_{0}$ is a priori estimate of $\mathrm{X}$, which is set to zero when no priori information is available. After solving for $\mathrm{X}$, we have voxel-wise maps of changes in concentration for each condition, participant, and chromophore.

The transformation matrices used to transform the subject sensitivity profiles to MNI space (see Section 2.3.3) were also applied to the voxel-wise maps for each of the conditions. The voxel-wise maps were intersected with the Group mask so that only those voxels contained within the Group mask would be included in the Group analyses.

\section{Group analyses}

Behavioral analyses. Accuracy (A') was calculated based on the hit (H) and false alarm (FA) rates using Aaronson and Watts' (Aaronson and Watts, 1987) updated version of Grier's formula (Grier, 1971) to compensate for below-chance performance:

If $\mathrm{H} \geq \mathrm{FA}: A^{\prime}=1 / 2+\left\{[(H-\mathrm{FA}) *(1+H-\mathrm{FA})] /\left[4^{*} H^{*}(1-\mathrm{FA})\right]\right\}$

If $\mathrm{H}<\mathrm{FA}: A^{\prime}=1 / 2-\left\{\left[(\mathrm{FA}-H)^{*}(1+\mathrm{FA}-H)\right] /\left[4 * \mathrm{FA}^{*}(1-H)\right]\right\}$

According to this formula, $\mathrm{A}^{\prime}$ of 1 indicates perfect performance and a score of 0.5 indicates chance performance. A' was calculated for each load and task for each of the younger and older participants. An ANOVA with one between-subjects factor (Age [younger and older]) and two within-subjects factors (Load [1, 2, 3] and Complexity [Color, Shape]) was run. Post-hoc comparisons with Bonferroni corrections were conducted on main effects and interactions that were significant.

Capacity (K) estimates were calculated for each load using Pashler's formula (the appropriate formula for a 'whole array' test) given by:

$K=$ load $*(H-\mathrm{FA}) /(1-\mathrm{FA})$;

From these estimates, the maximum capacity (max K) across loads for each participant for each task was extracted. An ANOVA with one between-subjects factor (Age) and one within-subjects factor (Complexity) was run on the max K values. Post-hoc comparisons with Bonferroni corrections were conducted on main effects and interactions that were significant.

fNIRS analyses. Only trials corresponding to correct responses were used for the analyses. All participants achieved an accuracy of $60 \%$ or greater across all the conditions. An ANOVA each with one between-subjects factor (Age) and three within-subjects factors (Load, Complexity, and Type [Same, Different]) were run separately for $\mathrm{HbO}$ and $\mathrm{HbR}$ concentration. The main effects and interactions from the two ANOVAs were corrected for familywise errors using 3dClustSim (corrected at alpha value of 0.05 , corresponding to a cluster size threshold of 24 voxels with a voxel resolution of $2 \times 2 \times 2 \mathrm{~mm}$ ).

We pooled the main effects and interactions from the two ANOVAs into 'Overall VWM' effects and 'Age-specific' effects. Overall VWM effects were those that did not have a main effect or an interaction of Age. These included the main effects of Load and Complexity and the interaction between Load and Complexity. Age-specific effects were those that showed any effect of Age and these included the main effect of Age and the 
interactions between Age and Complexity, Age and Load, and Age, Load and Complexity.

For overall VWM effects and age-specific effects, voxels were further classified such that they belonged to only one type of effect. The rationale for this step was to ensure that the highest-level interaction effect was given priority. The criteria used were: (1) if the voxel contributed to a three-way and a two-way interaction effect, it was classified as belonging to the three-way interaction. For example, if a voxel showed an Age $\times$ Load $\times$ Complexity effect as well as a significant Age $x$ Load interaction, that voxel was labelled as an Age $\times$ Load $\times$ Complexity effect. (2) If a voxel had 2 two-way interactions, it was classified as belonging to the interaction based on the priority of our questions in the present study, with Age effects receiving top priority, Load receiving second priority, and Complexity receiving third priority. For example, if a voxel had significant Age $\times$ Complexity interaction and an Age $\times$ Load interaction, it was classified as an Age $\times$ Load voxel. Our analyses focussed on $\mathrm{HbO}$ age-specific effects. All other effects are reported in the Supplementary material.

\section{Results}

\section{Behavioral results}

\section{Accuracy (A')}

Fig. 3 shows mean and SEM for each Age, Load and Complexity. Analysis of variance on A' revealed main effects of Age $\left(\mathrm{F}_{(1,46)}=37 ; \mathrm{p}<\right.$ $0.001)$, Load $\left(\mathrm{F}_{(2,71)}=151 ; \mathrm{p}<0.001\right)$ and Complexity $\left(\mathrm{F}_{(1,46)}=159 ; \mathrm{p}<\right.$ $0.001)$, as well as significant interactions between Age and Load $\left(\mathrm{F}_{(2,71)}=11 ; \mathrm{p}<0.001\right)$, Load and Complexity $\left(\mathrm{F}_{(2,79)}=43 ; \mathrm{p}<0.001\right)$, Age and Complexity $\left(\mathrm{F}_{(1,46)}=6 ; \mathrm{p}<0.001\right)$ and Age, Load, and Complexity $\left(\mathrm{F}_{(2,79)}=6 ; \mathrm{p}<0.01\right)$. Pairwise comparisons between levels of Load in the three-way interaction revealed that younger adults showed decreasing A' with increasing load only in the complex task ( $p$ $<0.001$ ). By contrast, older adults showed decreasing A' with increasing load in both tasks $(\mathrm{p}<0.005)$. Pairwise comparisons between levels of Complexity revealed that younger adults had greater A' for the simple than the complex task only at loads 2 and $3(\mathrm{p}<0.001)$. However, older adults had greater A' for the simple than the complex task at all loads $(\mathrm{p}<0.005)$. Finally, pairwise comparisons between levels of Age revealed that younger adults showed greater A' than older adults at loads 2 and 3 for the simple task $(\mathrm{p}<0.01)$. In the complex task, younger adults showed greater A' than older adults across all loads.

For max K, the main effects of Age $\left(\mathrm{F}_{(1,46)}=1774 ; \mathrm{p}<0.001\right)$ and Complexity $\left(\mathrm{F}_{(1,46)}=13 ; \mathrm{p}<0.002\right)$ were significant. Post-hoc comparisons revealed that the younger participants had greater max $\mathrm{K}$ values than older participants (younger $=2.8 \pm 0.08$ items; old $=2.3 \pm 0.08$ items). Overall, both groups showed greater max $\mathrm{K}$ values for the color than the shape task $($ color $=2.8 \pm 0.05$ items; shape $=2.3 \pm 0.1$ items $)$.

\section{fNIRS correspondence with $f M R I$ literature}

$\mathrm{HbO}$ and $\mathrm{HbR}$ effects from the current study were intersected with thirty VWM ROIs from previous fMRI studies (pooled together from Wijeakumar et al. (2015) and Ambrose et al. (2016)) to explore the correspondence between our fNIRS data and the fMRI literature. We summarize these effects here. Full details can be found in Supplementary Table 1. Supplemental Figure 1 shows a montage of the overlap between some ROIs and clusters of activation from the current study. Out of the thirty ROIs, eleven overlapped with clusters of activation that were significant here (labelled as 'Intersect') and seven were close to clusters of activation that were significant here (labelled as 'Adjacent'). These regions included the right superior intraparietal sulcus (sIPS), right IPS, bilateral anterior IPS (aIPS), bilateral ventral occipital cortex (VOC), bilateral dorsolateral prefrontal cortex (DLPFC), right frontal eye fields (FEF), bilateral occipital cortex (OCC), bilateral temporo-parietal junction (TPJ), bilateral inferior occipital gyrus (IOGf), right V3A and bilateral face-selective middle fusiform gyrus (fsMFG). Five of the remaining twelve ROIs shared the same MNI Atlas label as clusters of activation that were significant here (labelled as 'Same label' in Supplementary Table 1). These included left superior frontal gyrus (SFG), bilateral inferior frontal gyrus (IFG) and bilateral middle frontal gyrus (MFG). Out of the seven remaining ROIs, left anterior cingulate cortex (ACC) and left intraparietal sulcus (IPS) was not covered by the NIRS probe we used (labelled as 'Not covered' in Supplementary Table 1) as reported by Wijeakumar et al. (2015). Furthermore, ROIs from Ambrose et al. (2016) that were not included in the original study by Wijeakumar et al. (2015) such as left V1, left V3A and bilateral MFGf were also not covered by the probe geometry.

\section{Effects of load and Complexity on HbO activation}

$\mathrm{HbO}$ activation from the main effects of Load and Complexity as well as the interaction between Load and Complexity are shown in Supplemental Table 2. As is shown in Supplemental Figure 4, clusters in the posterior cortex such as left angular gyrus (AG) and left middle temporal gyrus (lMTG) showed increasing activation with increasing load in the simple task consistent with previous findings (Todd and Marois, 2005, 2004). In addition, lAG and lMTG showed a decrease in activation at the highest memory load for the complex task. These results closely match findings from our previous fMRI VWM study (Ambrose et al., 2016).

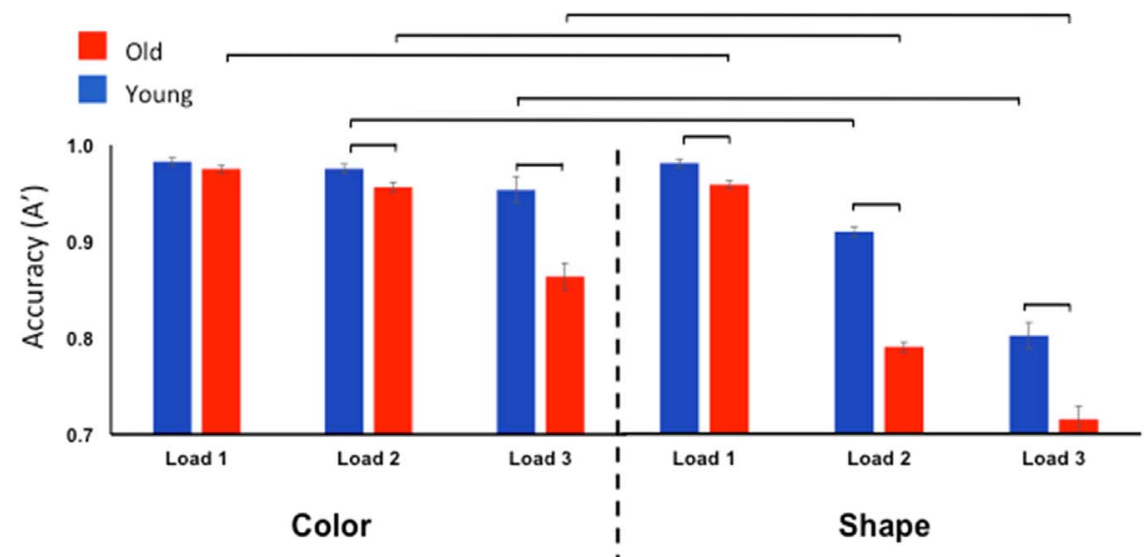

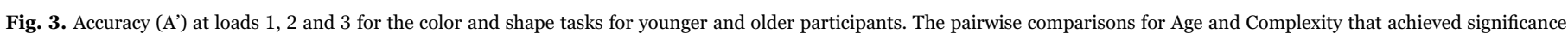
have been highlighted using '-'. Refer to text for pairwise comparisons between levels of Load that achieved significance. 
Table 1

Main effects and interactions for age-specific effects on $\mathrm{HbO}$ activation. Note than $\mathrm{F}$-stat values reported are prior to voxel classification described in Section 2.3.5.1.

\begin{tabular}{|c|c|c|c|c|c|c|c|}
\hline \multirow[t]{2}{*}{ Region } & \multirow[t]{2}{*}{ Hemi } & \multicolumn{3}{|c|}{ Center of Mass } & \multirow[t]{2}{*}{ Size $\left(\mathrm{mm}^{3}\right)$} & \multicolumn{2}{|l|}{ F-stat } \\
\hline & & $-\mathbf{x}$ & $\mathbf{y}$ & $\mathbf{z}$ & & -Mean & SEM \\
\hline \multicolumn{8}{|l|}{ Age } \\
\hline IFG & $\mathrm{R}$ & -44.9 & -38.6 & 10 & 3488 & 6.4 & 0.15 \\
\hline IFG & $\mathrm{L}$ & 51.8 & -26 & 10.6 & 1024 & 4.9 & 0.05 \\
\hline AG & $\mathrm{L}$ & 46.7 & 69.6 & 26.9 & 432 & 6.3 & 0.2 \\
\hline ITG & $\mathrm{R}$ & -59.9 & 54.7 & -6.3 & 360 & 4.5 & 0.06 \\
\hline MTG & $\mathrm{R}$ & -53.2 & 74.5 & 12.1 & 232 & 5.3 & 0.16 \\
\hline MTG & $\mathrm{L}$ & 48 & 63.6 & 20.7 & 144 & 6.3 & 0.21 \\
\hline MTG & $\mathrm{R}$ & -47.2 & 70 & 2.5 & 64 & 6.3 & 0.21 \\
\hline MOG & $\mathrm{R}$ & -54 & 75.3 & -0.7 & 24 & 5.3 & 0.16 \\
\hline ITG & $\mathrm{R}$ & -54 & 72 & -5 & 16 & 5.3 & 0.16 \\
\hline \multicolumn{8}{|c|}{ Age $\times$ Load } \\
\hline MOG & $\mathrm{L}$ & 45.2 & 78.3 & 1.2 & 1208 & 4.1 & 0.05 \\
\hline STG & $\mathrm{R}$ & -63.7 & 41.9 & 13.7 & 920 & 3.7 & 0.05 \\
\hline PostCG & $\mathrm{R}$ & -51 & 25.6 & 51.4 & 616 & 3.8 & 0.03 \\
\hline IOG & $\mathrm{R}$ & -46 & 78 & -9.7 & 432 & 3.3 & 0.01 \\
\hline IFG & $\mathrm{L}$ & 54.3 & -18.1 & 19.9 & 312 & 3.7 & 0.1 \\
\hline SMG & $\mathrm{R}$ & -54.7 & 31.5 & 40.9 & 264 & 3.4 & 0.04 \\
\hline \multicolumn{8}{|c|}{ Age $\times$ Complexity } \\
\hline IPL & $\mathrm{R}$ & -44.4 & 54 & 45.8 & 488 & 4.7 & 0.03 \\
\hline SMG & $\mathrm{L}$ & 65.7 & 37.7 & 24.9 & 56 & 4.3 & 0.04 \\
\hline \multicolumn{8}{|c|}{ Age $\times$ Load $\times$ Complexity } \\
\hline IPL & $\mathrm{R}$ & -48.2 & 54.4 & 45.4 & 1624 & 3.8 & 0.04 \\
\hline MFG & $\mathrm{R}$ & -34.5 & -46.4 & 19.4 & 1320 & 4.2 & 0.06 \\
\hline MOG & $\mathrm{L}$ & 48.8 & 73.2 & -2 & 1080 & 3.7 & 0.03 \\
\hline SMG & $\mathrm{L}$ & 55.2 & 42.7 & 23.6 & 1072 & 3.9 & 0.06 \\
\hline MTG & $\mathrm{R}$ & -56.4 & 63.9 & -0.6 & 656 & 3.6 & 0.05 \\
\hline MTG & $\mathrm{L}$ & 61.2 & 49.1 & 10.2 & 320 & 3.8 & 0.08 \\
\hline IPL & $\mathrm{L}$ & 51.6 & 47.1 & 47.2 & 304 & 3.6 & 0.07 \\
\hline MFG & $\mathrm{L}$ & 44.4 & -11.8 & 41.6 & 288 & 4.3 & 0.14 \\
\hline SMG & $\mathrm{L}$ & 63.4 & 43.8 & 28.6 & 160 & 3.9 & 0.06 \\
\hline
\end{tabular}

\section{Age-specific fNIRS effects}

Age-specific effects included the main effect of Age and interactions between Age and Load, Age and Complexity, and Age, Load and Complexity. The clusters with significant differences in $\mathrm{HbO}$ activation for each effect are shown in Table 1. We focus on HbO activation here since it has been more widely discussed in the fNIRS literature. Full details of significant HbR effects can be found in the Supplemental material.

\section{Main effect of Age}

A main effect of Age was observed in several clusters across the frontal, temporal, parietal, and occipital cortices (see Fig. 4). Specifically, clusters in the posterior cortex such as right inferior temporal gyrus (rITG), right middle temporal gyrus (rMTG), and right middle occipital gyrus (rMOG) showed greater activation for the younger than the older adults. However, clusters in the anterior cortex such as the right inferior frontal gyrus (rIFG) showed greater activation for the older than for the younger adults. These effects are consistent with the PASA hypothesis. Note that two clusters in the posterior cortex - IAG and lMTG (shown in blue in Fig. 4) - showed greater activation in older adults. It is possible that these regions represent hubs that functionally bridge the posterior and anterior cortices.

\section{Interaction between Age and Load}

There was a significant interaction between Age and Load in feature-selective regions in the occipito-temporo-parietal cortices including lMOG, rSTG, rIOG and right supramarginal gyrus (rSMG; see Fig. 5). Younger adults showed increasing activation with increasing load as in prior studies. By contrast, older adults showed overactivation at the lowest load followed by a drop in activation with increasing load. This trend in activation is consistent with the CRUNCH hypothesis. Interestingly, these effects were also observed in motor and executive control areas such as the postCG and IFG (see Table 1) implying the spread of age-related deficits to higher order centres.

\section{Interaction between Age and Complexity}

An interaction between Age and Complexity was observed in IPL and ISMG (see Fig. 6). Older adults showed lesser activation than younger adults in the simple task. On the other hand, they showed greater activation than the younger adults in the complex task suggesting the employment of adaptive strategies to cope with the increased difficulty of the task due to the perceptual complexity.

\section{Interaction between Age, Load, and Complexity}

From the behavioral results, it is clear that older adults showed a reduction in VWM capacity for the complex task relative to the simple task. Moreover, from the main effect of Age (Section Main effect of Age), it is clear that PASA generalized to VWM processing in the present study. Following from these observations, the central question was whether the brain response to the manipulations of perceptual complexity and load were selective to the posterior cortex, to the anterior cortex, or general across both cortices. The Age $\times$ Load and Age $\times$ Complexity effects were generally isolated to the posterior cortex with one notable exception (IIFG). We consider the Age $\times$ Load $\times$ Complexity results in this context (see Fig. 7).

Modulation of the posterior cortex. In the posterior cortex, the interaction between Age, Load and Complexity was observed in clusters in bilateral IPL, bilateral MTG, IMOG and ISMG (see exemplar in left subplot in Fig. 7). In the simple task, younger adults showed increasing activation with increasing load, consistent with previous fMRI VWM findings (Todd and Marois, 2005, 2004; Ambrose et al., 2016). Younger adults in the complex task showed decreasing activation with increasing load. This is also consistent with the precipitous decline we observed at the highest load in our previous fMRI study (Ambrose et al., 2016). These data suggest participants had difficulty encoding and/or maintaining representations of the complex shape stimuli at the highest load.

Older adults in the simple task showed a similar trend in activation observed for the younger adults in the complex task (see Fig. 7). In particular, as the load was increased, there was a decrease in activation (see also, Mattay et al., 2006; Quinn and Eimas, 1998; Rypma et al., 2002), suggesting that older adults had difficulty encoding and/or maintaining representations even in the simple color task. Note that activation at the lowest load was greater for older than for younger adults, even though both groups had comparable behavioral performance (refer to Section Behavioral Results). These findings are in line with other reports of greater activation in older than younger adults at the lowest load (Mattay et al., 2006; Missonnier et al., 2011; Nagel et al., 2011; Nyberg et al., 2009; Schneider-Garces et al., 2010).

Interestingly, older adults in the complex task showed increasing activation with increasing load. This increase with load might be a result of older adults using different encoding and/or maintenance strategies than the younger adults to process the more complex stimuli. Indeed, during debriefing, younger adults reported using a 'gestalt' sense of the presentation arrays for both the simple and complex arrays, while older adults reported using naming strategies to compare the complex arrays, associating labels such as 'pork chop', 'rabbit', 'airplane' and 'state of Massachusetts' with individual stimuli. The use of such strategies is not uncommon in the ageing literature (Mattay et al., 2006; Paxton et al., 2008; Reuter-Lorenz and Campbell, 2008). This interpretation is consistent with the recruitment of SMG which is activated in verbal working memory studies (Awh et al., 1999, 1996a; Barch and Csernansky, 2007; Deschamps et al., 2014).

Modulation of the anterior cortex. In the anterior cortex, an 


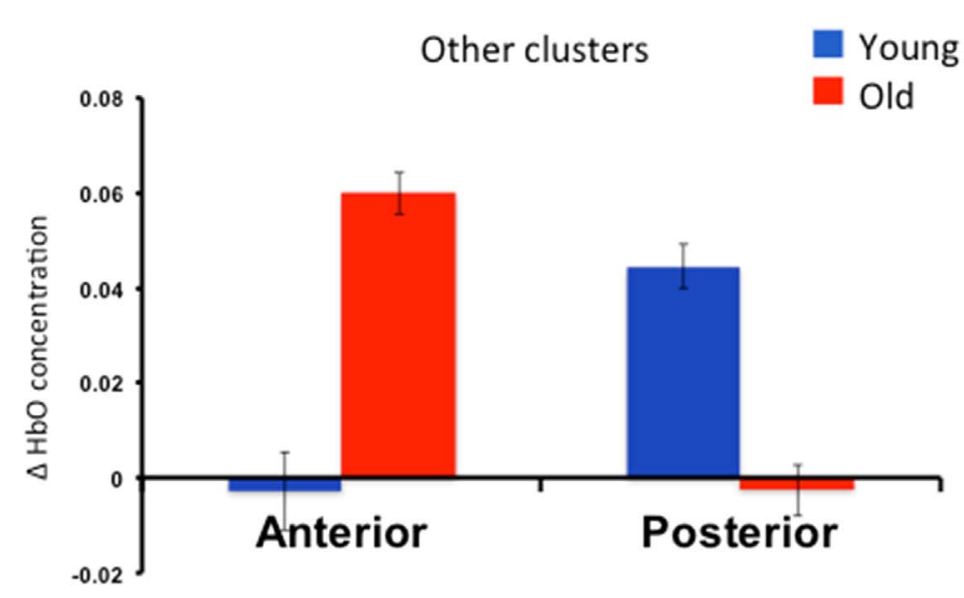

IAG /IMTG
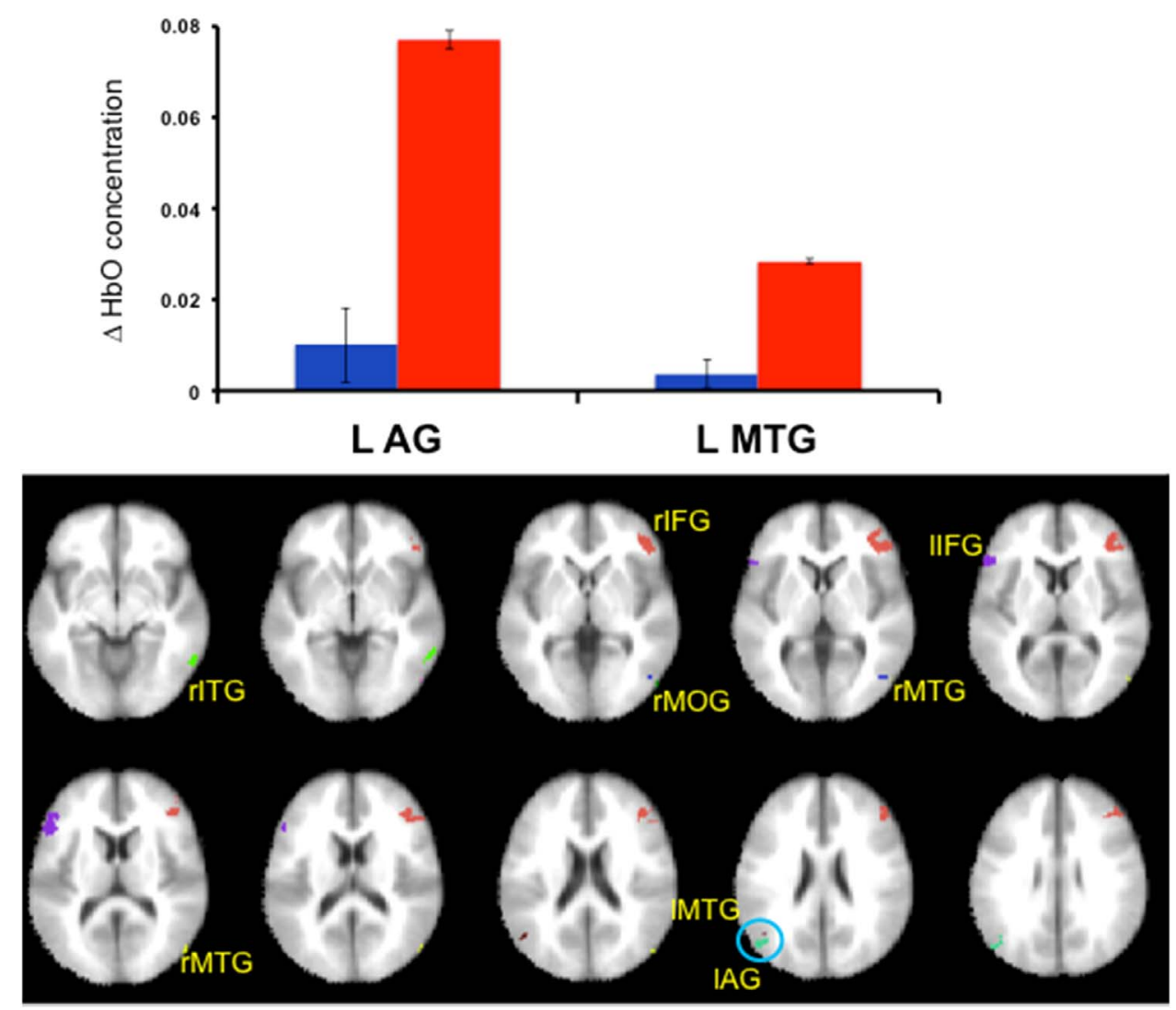

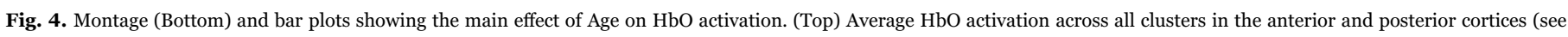

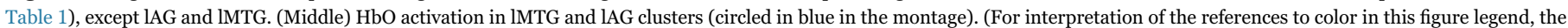
reader is referred to the web version of this article.)

interaction between Age, Load and Complexity was observed in bilateral MFG. For both age groups, the pattern in lMFG across the simple and complex tasks was consistent with the 3-way interactions in posterior cortex (see exemplary subplot in the left panel of Fig. 7).

In rMFG, however, there was a different pattern. For young adults in the simple task, there was a decrease in activation in rMFG (see right subplot in Fig. 7). In the complex task, young adults showed the opposite pattern with an increase in activation with increasing load. This latter effect suggests that rMFG may play a modulatory role, providing top-down input in the most challenging condition. For older adults in the simple task, rMFG did not show any change with increasing load. This might reflect top-down input across loads as older adults try to maintain VWM performance. In the complex task, however, there was high activation at the lowest load, and a steep decline in activation at the highest load, reminiscent of young adults' performance in Ambrose et al. (2016). At the lowest load this result might, once again, reflect top-down input from rMFG as older adults try to maintain reasonable performance in the complex task; the highest load, by contrast, might be well-beyond the compensatory range of rMFG as both activation and behavioral performance shows a sharp decline.

\section{Discussion}

The objective of the current study was to understand the nature of the shift in activation from the posterior to the anterior cortices in the ageing adult. For instance, recent reviews of PASA have suggested that 

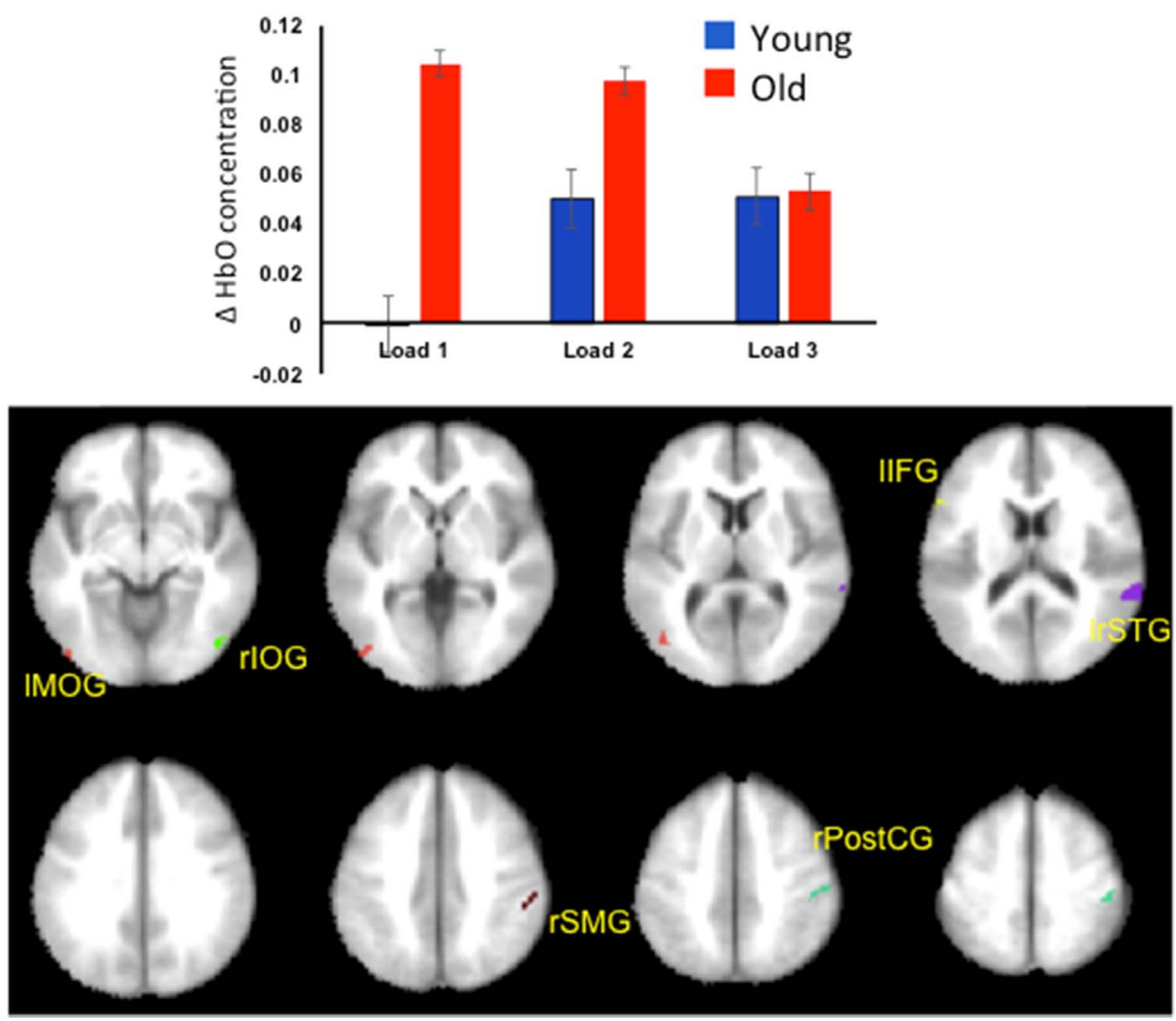

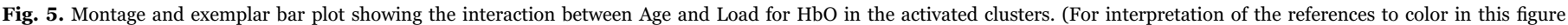
legend, the reader is referred to the web version of this article.)

age-related decline in perceptual processing in the posterior cortex might lead to increased compensatory activation in higher-order anterior processing centres. Alternatively, age-related decline in anterior cortex might result in over activity and impoverished top-down input leading to processing deficits in posterior cortex (see Monge and Madden, 2016 for review). Here, we asked whether modulating perceptual complexity and working memory load in two variants of a visual working memory task would differentially modulate activation across the anterior and posterior cortices in older compared to younger adults.

fNIRS reveals comparable modulation of VWM across load and complexity relative to $\mathrm{fMRI}$

To investigate these questions, we used a recently validated methodology with fNIRS to obtain voxel-wise measures of brain activation, enabling direct comparisons with the fMRI literature (Wijeakumar et al., 2017, 2015). Previous work has used fNIRS to explore the neural mechanisms that underlie age-related decline in working memory (Vermeij et al., 2016, 2014, 2012). However, these studies relied on recordings from two channels placed over the prefrontal cortex only and did not explore posterior cortical activity. In the current study, we concurrently record from the anterior and posterior cortices.

The clusters of activation from the current study overlapped with ROIs from previous VWM studies, providing validation for the use of fNIRS and our methodological pipeline to move analyses from conventional channel-space to voxel-space. Further, overall VWM effects were comparable to trends reported in previous fMRI VWM studies. Specifically, feature-selective regions showed an increase in activation with increasing working memory load for the simple color task. These findings are in agreement with previous studies that have shown that the extra-striate cortex maintains stimulus-specific representations
(D’Esposito and Postle, 2015; Postle, 2015; Shafritz and Marois, 2002; Todd and Marois, 2005, 2004). We also found decreasing activation in the complex task in clusters in the posterior cortex. These patterns resembled the trends we observed in our previous fMRI study (Ambrose et al., 2016). In the complex task, we suggest that increasing competition between metrically similar shape stimuli as the memory load increases leads to an inability to simultaneously maintain multiple WM representations. As a consequence, fewer items are actively maintained at high loads, leading to a decline in neural activation.

Posterior to anterior shift in ageing generalizes to VWM processing

PASA is a common finding across age-related cognitive studies (Anderson, 2000; Cabeza et al., 2004; Davis et al., 2008; Dennis et al., 2007; Grossman et al., 2002; Gutchess, 2014; Reuter-Lorenz et al., 2000; Reuter-Lorenz and Campbell, 2008; Reuter-Lorenz and Lustig, 2005; Rypma and D'Esposito, 2000). In the current study, we observed reduced activation in the posterior cortex in older adults in the lAG, rITG, MTG, rMOG, and rITG, suggesting a general decline in perceptual processing and/or maintenance of WM representations. There are a few possible reasons why the quality and/or quantity of perceptual / WM representations might be weakened. With senescence, the structure of the eye undergoes physiological changes such as the increase in density and hardening of the crystalline lens and reduction in the number of receptor cells. Further, there is a marked drop in visual acuity and contrast sensitivity (for review, see Monge and Madden, 2016). Electrophysiological studies in aged monkeys show a reduction in V1 synapses and the degradation of myelinated fibres, which results in an increase in the latency of passing visual information to higher order areas (Wang et al., 2005). Recent fNIRS studies also provided evidence to this effect at the cortical level by observing diminished $\mathrm{HbO}$ activation in older adults in response to a simple visual stimulus such as a reversing 


\section{Young \\ Old}
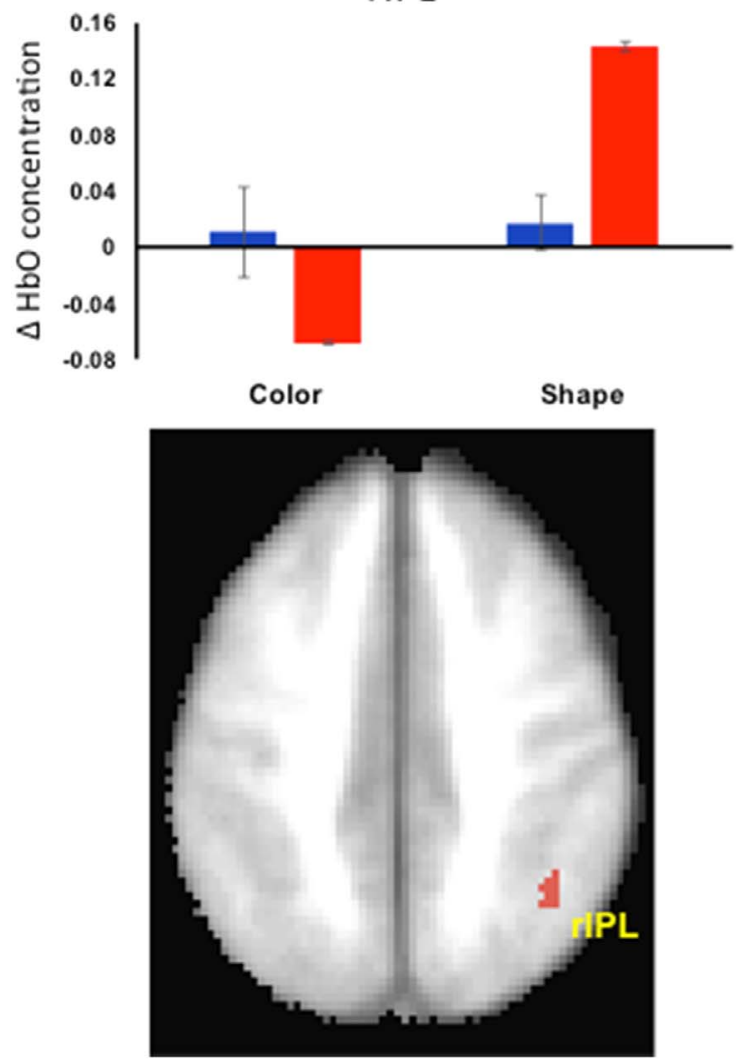

ISMG
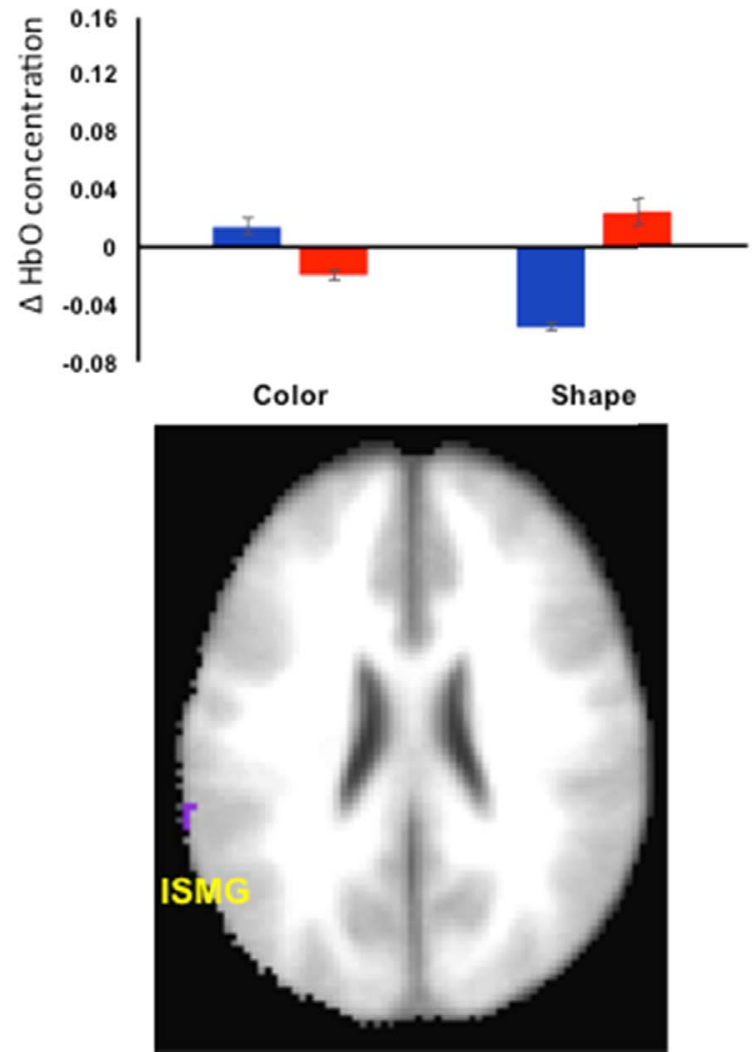

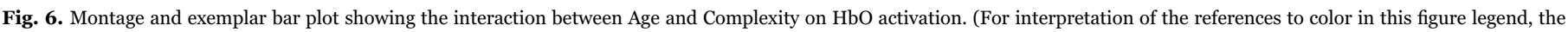
reader is referred to the web version of this article.)

checkerboard (Fabiani et al., 2014; Ward et al., 2015). It is possible that such long-term perceptual deficits might affect the 'baseline' activation of the ageing posterior cortex. Such perceptual deficits might also have effects on higher-order centres in the anterior cortex.

The anterior cortex is a commonly targeted site for studying age- related cognitive decline because it undergoes rapid loss in gray and white matter (Richards et al., 2016; Salat et al., 2004). Consistent with PASA, we found increased activation in the anterior cortex in IFG, particularly at low loads. This might reflect over activity as the anterior cortex tries to extract relevant information from poor perceptual / WM
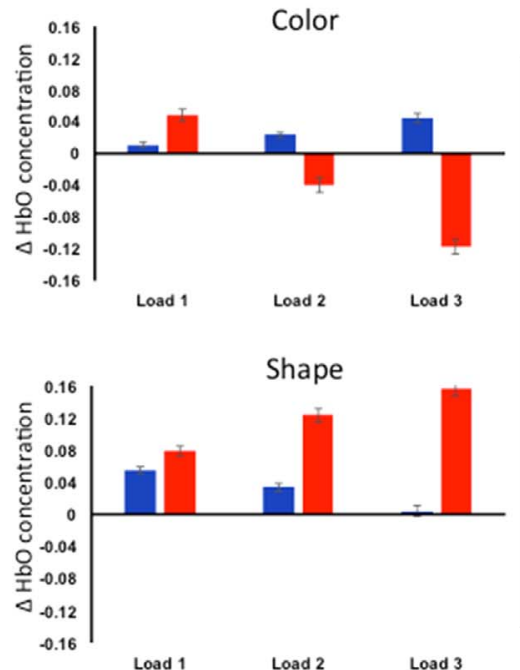
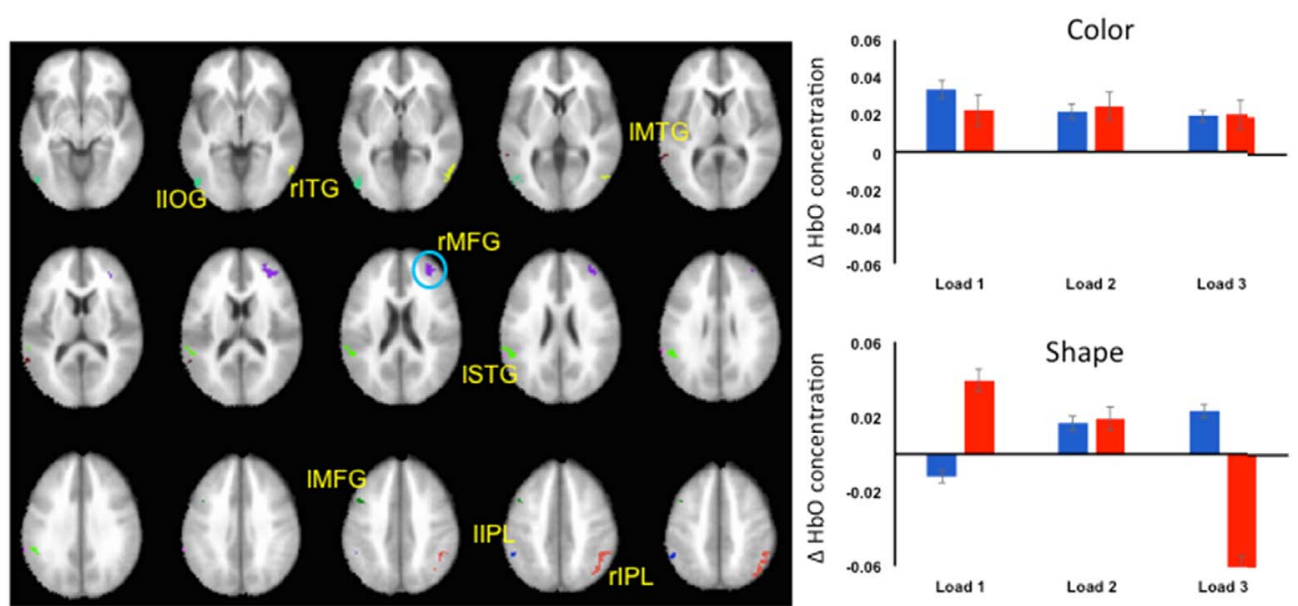

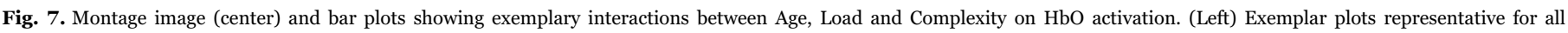

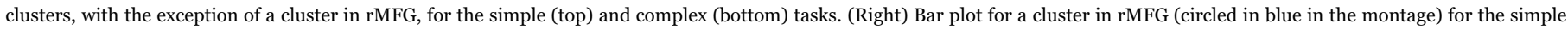
(top) and complex (bottom) tasks. (For interpretation of the references to color in this figure legend, the reader is referred to the web version of this article.) 
representations to make a timely decision. Alternatively, the frontal cortex might be trying to boost WM representations in the posterior cortex to compensate for age-related decline in posterior regions.

Differential modulation of posterior and anterior cortices in younger and older adults

The central question in this study was how these age-related changes in posterior and anterior activation might be differentially modulated by the task demands. Overall, we found modulation of the posterior and anterior cortices in both the younger and older adults as load and complexity were varied. However, the pattern of modulation differed between age groups.

Younger adults showed increasing activation with increasing load in the simple task in parts of the posterior cortex such as the IPL, consistent with previous VWM studies (Linden et al., 2003; Shafritz and Gore, 2002; Todd and Marois, 2005, 2004; Xu and Chun, 2006). The IPL is well known for its role in spatial attention, and activation in the IPL might be linked to covertly attending to multiple objects (Linden et al., 2003). Regions proximal to the IPL also have functional relevance to working memory. For example, the intraparietal sulcus, which is dorsal to IPL, plays a key role in the maintenance of items in VWM (Xu and Chun, 2006). For instance, activation in the intraparietal sulcus increases up to the capacity of the VWM system and plateaus thereafter (Todd and Marois, 2005, 2004; Ambrose et al., 2016). By contrast, a region superior to the IPL has been shown to be more selective to complexity, but not the quantity of items being encoded / remembered ( $\mathrm{Xu}$ and Chun, 2006).

In contrast to results from the simple task, we found that increasing load in the complex task resulted in decreasing activation in IPL, lMOG, ISMG, and MTG. As suggested above, we contend that the decrease in activation at higher load reflects the inability to encode and/or simultaneously maintain representations of multiple metrically-similar shape stimuli. This is consistent with previous work from our lab showing a precipitous decline in neural activation at high load with the same shape stimuli (Ambrose et al., 2016). Similarly, Xu and Chun (2006) observed that activation differed from load 2 to 6 in the superior intraparietal sulcus and lateral occipital complex for simple but not for the complex shapes that they used (Xu and Chun, 2006).

The anterior cortex of younger adults was also modulated by perceptual complexity and load. In particular, lMFG followed the pattern evident in areas of the posterior cortex, for instance, IPL; however, rMFG showed a different pattern. Most notably, there was an increase in rMFG activation over load for the complex task. This is similar to what was reported by Edin et al. (2009). These researchers have proposed that dorsolateral PFC increases baseline activation of posterior WM areas at high loads to help stabilize WM representations (Edin et al., 2009). It is possible that rMFG is serving this function for young adults in the present study, increasing top-down input to aid the posterior cortex in the most challenging conditions. Taken together, then, younger adults showed modulation of both the posterior and anterior cortex with varying complexity and load.

Older adults also showed modulation of the posterior and anterior cortices but in a different manner relative to younger adults. In the simple task, older adults showed the same trend in activation as the younger adults in the complex task, with high activity at low loads and a decline in activation at higher loads. Thus, consistent with behavioral results showing poorer performance in the color task, older adults appear to work harder at the neural level to remember even simple featured objects. Notably, older adults showed greater activation in response to a load of 1 item in the simple task than younger adults despite matched behavioral performance. It is possible this reflects the degradation of the precision of perceptual and/or WM representations which occurs through the broadening of tuning curves (Reuter-Lorenz and Lustig, 2005) and/or increased perceptual noise (Cerella, 1985; Li et al., 2001; Salthouse, 1996; Salthouse and Meinz, 1995). This decline in perceptual / WM representations might then necessitate overactivation in the posterior cortex.

A different form of decline was evident in the complex task with increasing load resulting in increasing activation in verbal working memory areas such as SMG. The SMG has been activated in previous verbal working memory studies (Awh et al., 1999, 1996b; Barch and Csernansky, 2007; Deschamps et al., 2014). For instance, in a recent study, SMG was examined using transcranial magnetic stimulation during a same/different judgment task with varying levels of phonological complexity and a verbal working memory task with varying levels of phonological complexity and working memory load. The researchers found that disruption of SMG only affected verbal working memory (Deschamps et al., 2014). In the current study, increasing SMG activation with increasing working memory load in the complex task might reflect the use of verbal strategies to encode each shape stimulus and associate it with a label. Note that, despite increasing reliance on verbal working memory, behavioral performance in older adults was much worse than younger adults for the complex task.

The observed decline in the posterior cortex was also accompanied by decline in the anterior cortex. In particular, IMFG showed the same pattern of over-activity at low load for the simple task and an increase in activation over load for the complex task. The latter effect suggests IMFG may play a role in verbal working memory or coupled to verbal working memory areas. Recall that we optimized the fNIRS probe geometry to record from VWM areas, not verbal WM areas. Thus, precisely how older adults are engaging verbal working memory in this task will require future examination.

As with the younger adults, we observed a different pattern of results in rMFG. Degradation of perceptual and/or maintenance processes in the posterior cortex might have elicited a top-down influence from the rMFG to boost performance for a load of 1 item in the simple task. However, this level of activation was maintained without any increase as behavioral performance declined with increasing load and posterior activation decreased. This result might be related to findings from Gazzaley et al. (2008) who have shown agerelated delays in top-down suppression of task-irrelevant information during the early stages of visual processing as well as poorer memory of task-relevant information. rMFG activity in the complex task might also reflect degraded top-down influences on VWM. Here we found robust activation at load 1 , but a precipitous decline in activation at higher loads as the task demands increased.

It is important to note that differences in activation in parts of the anterior cortex might also reflect the differing effort needed to extract information from poorer posterior representations. In the current study, these two processes might be inextricably linked. Previous studies have, however, reported effects from top-down modulation of control on encoding, maintenance and retrieval processes in working memory (see Gazzaley and Nobre (2012) for a review, Zanto et al., 2011). Future work should explore the possibility of teasing apart topdown modulation from the anterior cortex trying to extract information necessary for decision-making from degraded representations.

\section{Summary}

In the present report, we found age-related decline in both anterior and posterior cortices, with task-specific modulation of activation throughout the VWM network. Older adults showed over-activity at low loads, particularly when they were asked to remember simple color stimuli, with a decline in activation at higher loads. By contrast, they showed an increase in activation with an increase in memory load for complex shape stimuli, possibly reflecting the involvement of verbal working memory processes. In addition, we found evidence of deficient top-down compensatory activation in rMFG. Considered together, the present study sheds new light on changes in posterior and anterior neural activity with ageing, showing pervasive decline throughout the VWM network that spans anterior and posterior cortical regions. We 
suggest that future work should continue to study age-related decline by comparing manipulations across different tasks with varying complexity to reveal how the entire VWM network is modulated at it tries to compensate for structural neural decline with aging.

\section{Acknowledgment}

SW acknowledges funding from an Interdisciplinary Research Grant from the DELTA Centre at the University of Iowa.

\section{Appendix A. Supporting information}

Supplementary data associated with this article can be found in the online version at doi:10.1016/j.neuroimage.2017.06.019.

\section{References}

Aaronson, D., Watts, B., 1987. Extensions of Grier's computational formulas for A' and B" to below-chance performance. Psychol. Bull. 102, 439-442.

Ambrose, J.P., Wijeakumar, S., Buss, A.T., Spencer, J.P., 2016. Feature-based change detection reveals inconsistent individual differences in visual working memory capacity. Front. Syst. Neurosci. 10, 1-10. http://dx.doi.org/10.3389/ fnsys.2016.00033.

Anderson, J.R., 2000. Cognitive Psychology and It Implications. Worth, New York.

Awh, E., Jonides, J., Smith, E.E., Schumacher, E.H., Koeppe, R.A., Katz, S., 1996a. Dissociation of storage and rehearsal in verbal working memory: evidence from Positron Emission Tomography. Psychol. Sci. 7, 25-31. http://dx.doi.org/10.1111/ j.1467-9280.1996.tb00662.x

Awh, E., Jonides, J., Smith, E.E., Schumacher, E.H., Koeppe, R.A., Katz, S., 1996b. Dissociation of storage and rehearsal in verbal working memory: evidence from Positron Emission Tomography. Psychol. Sci. 7, 25-31. http://dx.doi.org/10.1111/ j.1467-9280.1996.tb00662.x.

Awh, E., Jonides, J., Smith, E.E., Buxton, R.B., Frank, L.R., Love, T., Wong, E.C., Gmeindl, L., 1999. Rehearsal in spatial working memory: evidence from neuroimaging. Psychol. Sci. 10, 433-437.

Barch, D.M., Csernansky, J.G., 2007. Abnormal parietal cortex activation during working memory in schizophrenia: verbal phonological coding disturbances versus domaingeneral executive dysfunction. Am. J. Psychiatry 164, 1090-1098. http://dx.doi.org/ 10.1176/appi.ajp.164.7.1090.

Bortfeld, H., Wruck, E., Boas, D. a., 2007. Assessing infants' cortical response to speech using near-infrared spectroscopy. Neuroimage 34, 407-415. http://dx.doi.org/ 10.1016/j.neuroimage.2006.08.010.

Cabeza, R., Daselaar, S.M., Dolcos, F., Prince, S.E., Budde, M., Nyberg, L., 2004. Taskindependent and task-specific age effects on brain activity during working memory, visual attention and episodic retrieval. Cereb. Cortex 14, 364-375. http:// dx.doi.org/10.1093/cercor/bhg133.

Calvetti, D., Morigi, S., Reichel, L., Sgallari, F., 2000. Tikhonov regularization and the Lcurve for large discrete ill-posed problems. J. Comput. Appl. Math. 123, 423-446. http://dx.doi.org/10.1016/S0377-0427(00)00414-3.

Cappell, K.A., Gmeindl, L., Reuter-Lorenz, P.A., 2010. Age differences in prefontal recruitment during verbal working memory maintenance depend on memory load. Cortex 46, 462-473. http://dx.doi.org/10.1016/j.cortex.2009.11.009.

Cerella, J., 1985. Information processing rates in the elderly. Psychol. Bull. 98, 67-83. http://dx.doi.org/10.1037/0033-2909.98.1.67.

Chen, L.C., Sandmann, P., Thorne, J.D., Herrmann, C.S., Debener, S., 2015. Association of concurrent fNIRS and EEG signatures in response to auditory and visual stimuli. Brain Topogr. 28, 710-725. http://dx.doi.org/10.1007/s10548-015-0424-8.

Cutini, S., Scatturin, P., Menon, E., Bisiacchi, P.S., Gamberini, L., Zorzi, M., Dell'Acqua, R., 2008. Selective activation of the superior frontal gyrus in task-switching: an event-related fNIRS study. Neuroimage 42, 945-955. http://dx.doi.org/10.1016/ j.neuroimage.2008.05.013.

D’Esposito, M., Postle, B.R., 2015. The cognitive neuroscience of working memory. Annu. Rev. Psychol. 66, 186-219. http://dx.doi.org/10.1093/acprof:oso/ 9780198570394.001.0001.

Davis, S.W., Dennis, N.A., Daselaar, S.M., Fleck, M.S., Cabeza, R., 2008. Que PASA? The posterior anterior shift in aging. Cereb. Cortex 18, 1201-1209. http://dx.doi.org/ 10.1093/cercor/bhm155.

Dennis, N.A., Daselaar, S., Cabeza, R., 2007. Effects of aging on transient and sustained successful memory encoding activity. Neurobiol. Aging 28, 1749-1758. http:// dx.doi.org/10.1016/j.neurobiolaging.2006.07.006.

Deschamps, I., Baum, S.R., Gracco, V.L., 2014. On the role of the supramarginal gyrus in phonological processing and verbal working memory: evidence from rTMS studies. Neuropsychologia 53, 39-46. http://dx.doi.org/10.1016/ j.neuropsychologia.2013.10.015.

Drucker, D.M., Aguirre, G.K., 2009. Different spatial scales of shape similarity representation in lateral and ventral LOC. Cereb. Cortex 19, 2269-2280. http:// dx.doi.org/10.1093/cercor/bhn244.

Druzgal, T.J., D’Esposito, M., 2003. Dissecting contributions of prefrontal cortex and fusiform face area to face working memory. J. Cogn. Neurosci. 15, 771-784. http:// dx.doi.org/10.1162/089892903322370708.
Edin, F., Klingberg, T., Johansson, P., McNab, F., Tegnér, J., Compte, A., 2009 Mechanism for top-down control of working memory capacity. Proc. Natl. Acad. Sci. 106, 6802-6807. http://dx.doi.org/10.1073/pnas.0901894106.

Fabiani, M., Gordon, B.A., Maclin, E.L., Pearson, M.A., Brumback-Peltz, C.R., Low, K.A., McAuley, E., Sutton, B.P., Kramer, A.F., Gratton, G., 2014. Neurovascular coupling in normal aging: a combined optical, ERP and fMRI study. Neuroimage 85, 592-607. http://dx.doi.org/10.1016/j.neuroimage.2013.04.113.

Fillmore, P.T., Phillips-Meek, M.C., Richards, J.E., 2015. Age-specific MRI brain and head templates for healthy adults from 20 through 89 years of age. Front. Aging Neurosci. 7. http://dx.doi.org/10.3389/fnagi.2015.00044.

Gagnon, L., Yücel, M.A., Dehaes, M., Cooper, R.J., Perdue, K.L., Selb, J., Huppert, T.J., Hoge, R.D., Boas, D.A., 2012. Quantification of the cortical contribution to the NIRS signal over the motor cortex using concurrent NIRS-fMRI measurements. Neuroimage 59, 3933-3940. http://dx.doi.org/10.1016/j.neuroimage.2011.10.054

Gazzaley, A., Nobre, A.C., 2012. Top-down modulation: bridging selective attention and working memory. Trends Cogn. Sci.. http://dx.doi.org/10.1016/j.tics.2011.11.014.

Gazzaley, A., Clapp, W., Kelley, J., McEvoy, K., Knight, R.T., D’Esposito, M., 2008. Agerelated top-down suppression deficit in the early stages of cortical visual memory processing. Proc. Natl. Acad. Sci. 105, 13122-13126. http://dx.doi.org/10.1073/ pnas.0806074105.

Grier, J.B., 1971. Nonparametric indexes for sensitivity and bias: computing formulas. Psychol. Bull. 75, 410-429.

Grossman, M., Cooke, A., DeVita, C., Alsop, D., Detre, J., Chen, W., Gee, J., 2002. Agerelated changes in working memory during sentence comprehension: an fMRI study. Neuroimage 15, 302-317. http://dx.doi.org/10.1006/nimg.2001.0971.

Gutchess, A., 2014. Plasticity of the aging brain: new directions in cognitive neuroscience. Science (80-. ) 346, 579-582. http://dx.doi.org/10.1126/ science.1254604.

Huppert, T.J., Hoge, R.D., Diamond, S.G., Franceschini, M.A., Boas, D.A., 2006. A temporal comparison of BOLD, ASL, and NIRS hemodynamic responses to motor stimuli in adult humans. Neuroimage 29, 368-382. http://dx.doi.org/10.1016/ j.neuroimage.2005.08.065.

Learmonth, A.E., Nadel, L., Newcombe, N.S., 2002. Children's use of landmarks: implications for modularity theory. Psychol. Sci. 13, 337-341.

Li, S.C., Lindenberger, U., Sikstr??m, S., 2001. Aging cognition: from neuromodulation to representation. Trends Cogn. Sci.. http://dx.doi.org/10.1016/S1364-6613(00) 01769-1.

Linden, D.E.J., Bittner, R.A., Muckli, L., Waltz, J.A., Kriegeskorte, N., Goebel, R., Singer, W., Munk, M.H.J., 2003. Cortical capacity constraints for visual working memory: dissociation of fMRI load effects in a fronto-parietal network. Neuroimage 20, 1518-1530. http://dx.doi.org/10.1016/j.neuroimage.2003.07.021.

Luck, S., Vogel, E., 1997. The capacity of visual working memory for features and conjunctions. Nature 390, 279-281.

Ma, W.J., Husain, M., Bays, P.M., 2014. Changing concepts of working memory. Nat. Neurosci. 17, 347-356. http://dx.doi.org/10.1038/nn.3655.

Mattay, V.S., Fera, F., Tessitore, A., Hariri, A.R., Berman, K.F., Das, S., MeyerLindenberg, A., Goldberg, T.E., Callicott, J.H., Weinberger, D.R., 2006. Neurophysiological correlates of age-related changes in working memory capacity. Neurosci. Lett. 392, 32-37. http://dx.doi.org/10.1016/j.neulet.2005.09.025.

Missonnier, P., Herrmann, F.R., Rodriguez, C., Deiber, M.P., Millet, P., Fazio-Costa, L. Gold, G., Giannakopoulos, P., 2011. Age-related differences on event-related potentials and brain rhythm oscillations during working memory activation. J. Neural Transm. 118, 945-955. http://dx.doi.org/10.1007/s00702-011-0600-2.

Monge, Z.A., Madden, D.J., 2016. Linking cognitive and visual perceptual decline in healthy aging: the information degradation hypothesis. Neurosci. Biobehav. Rev. 69, 166-173. http://dx.doi.org/10.1016/j.neubiorev.2016.07.031.

Nagel, I.E., Preuschhof, C., Li, S.-C., Nyberg, L., Bäckman, L., Lindenberger, U., Heekeren, H.R., 2011. Load modulation of BOLD response and connectivity predicts working memory performance in younger and older adults. J. Cogn. Neurosci. 23, 2030-2045. http://dx.doi.org/10.1162/jocn.2010.21560.

Nyberg, L., Dahlin, E., Stigsdotter Neely, A., BÄckman, L., 2009. Neural correlates of variable working memory load across adult age and skill: dissociative patterns within the fronto-parietal network: cognition and Neurosciences. Scand. J. Psychol. 50, 41-46. http://dx.doi.org/10.1111/j.1467-9450.2008.00678.x.

Paxton, J.L., Barch, D.M., Racine, C.A., Braver, T.S., 2008. Cognitive control, goal maintenance, and prefrontal function in healthy aging. Cereb. Cortex 18, 1010-1028 . http://dx.doi.org/10.1093/cercor/bhm135.

Pessoa, L., Ungerleider, L., 2004. Neural correlates of change detection and change blindness in a working memory task. Cereb. Cortex 14, 511-520.

Plichta, M.M., Gerdes, A.B.M., Alpers, G.W., Harnisch, W., Brill, S., Wieser, M.J., Fallgatter, A.J., 2011. Auditory cortex activation is modulated by emotion: a functional near-infrared spectroscopy (fNIRS) study. Neuroimage 55, 1200-1207. http://dx.doi.org/10.1016/j.neuroimage.2011.01.011.

Postle, B.R., 2015. The cognitive neuroscience of visual short-term memory. Curr. Opin. Behav. Sci. 1, 40-46. http://dx.doi.org/10.1016/j.cobeha.2014.08.004.

Quinn, P.C., Eimas, P.D., 1998. Evidence for a global categorical representation for humans by young infants. J. Exp. Child Psychol. 69, 151-174.

Reuter-Lorenz, P.A., Campbell, K.A., 2008. Neurocognitive ageing and the Compensation Hypothesis. Curr. Dir. Psychol. Sci. 17, 177-182.

Reuter-Lorenz, P.A., Lustig, C., 2005. Brain aging: reorganizing discoveries about the aging mind. Curr. Opin. Neurobiol.. http://dx.doi.org/10.1016/j.conb.2005.03.016.

Reuter-Lorenz, P.A., Jonides, J., Smith, E.E., Hartley, A., Miller, A., Marshuetz, C., Koeppe, R.A., 2000. Age differences in the frontal lateralization of verbal and spatial working memory revealed by PET. J. Cogn. Neurosci. 12, 174-187. http:// dx.doi.org/10.1162/089892900561814.

Richards, J.E., Xie, W., 2015. Brains for all the ages. Adv. Child Dev. Behav., 1-52. 
http://dx.doi.org/10.1016/bs.acdb.2014.11.001.

Richards, J.E., Sanchez, C., Phillips-Meek, M., Xie, W., 2016. A database of ageappropriate average MRI templates. Neuroimage 124, 1254-1259. http:// dx.doi.org/10.1016/j.neuroimage.2015.04.055.

Rodrigo, A.H., Di Domenico, S.I., Ayaz, H., Gulrajani, S., Lam, J., Ruocco, A.C., 2014. Differentiating functions of the lateral and medial prefrontal cortex in motor response inhibition. Neuroimage 85, 423-431. http://dx.doi.org/10.1016/ j.neuroimage.2013.01.059.

Rypma, B., D’Esposito, M., 2000. Isolating the neural mechanisms of age-related changes in human working memory. Nat. Neurosci. 3, 509-515. http://dx.doi.org/10.1038/ 74889.

Rypma, B., Berger, J.S., D’Esposito, M., 2002. The influence of working-memory demand and subject performance on prefrontal cortical activity. J. Cogn. Neurosci. 14, 721-731. http://dx.doi.org/10.1162/08989290260138627.

Salat, D.H., Buckner, R.L., Snyder, A.Z., Greve, D.N., Desikan, R.S.R., Busa, E., Morris, J.C., Dale, A.M., Fischl, B., 2004. Thinning Cereb. Cortex Aging, 721-730. http:// dx.doi.org/10.1093/cercor/bhh032.

Salthouse, Timothy A., 1994. The aging of working memory. Neuropsychology 8 (4), 535-543. http://dx.doi.org/10.1037/0894-4105.8.4.535, (Working Memory).

Salthouse, Timothy A., 1996. The processing-speed theory of adult age differences in cognition. Psychol. Rev. 103, 403-428. http://dx.doi.org/10.1037/0033295X.103.3.403.

Salthouse, T.A., Meinz, E.J., 1995. Aging, inhibition, working memory, and speed. J. Gerontol. - Ser. B Psychol. Sci. Soc. Sci. 50 B, P297-P306. http://dx.doi.org/ 10.1093/geronb/50B.6.P297.

Schneider-Garces, N.J., Gordon, B.A., Brumback-Peltz, C.R., Shin, E., Lee, Y., Sutton, B.P., Maclin, E.L., Gratton, G., Fabiani, M., 2010. Span, CRUNCH, and beyond: working memory capacity and the aging brain. J. Cogn. Neurosci. 22, 655-669. http://dx.doi.org/10.1162/jocn.2009.21230.

Shafritz, Marois, G., 2002. The role of parietal cortex in visual feature binding. PNAS 99, 10917-10922.

Shafritz, K.M., Gore, J.C., 2002. The role of the parietal cortex in. PNAS 99, 10917-10922.

Todd, J.J., Marois, R., 2004. Capacity limit of visual short-term memory in human posterior parietal cortex. Nature 428, 751-754.

Todd, J.J., Marois, R., 2005. Posterior parietal cortex activity predicts individual differences in visual short-term memory capacity. Cogn. Affect. Behav. Neurosci. 5,
144-155.

Vermeij, A., van Beek, A.H.E.A., Olde Rikkert, M.G.M., Claassen, J.A.H.R., Kessels, R.P.C., 2012. Effects of aging on cerebral oxygenation during working-memory performance: a functional near-infrared spectroscopy study. PLoS One 7, 1-11. http://dx.doi.org/10.1371/journal.pone.0046210.

Vermeij, A., van Beek, A.H.E.A., Reijs, B.L.R., Claassen, J.A.H.R., Kessels, R.P.C., 2014 An exploratory study of the effects of spatial working-memory load on prefrontal activation in low- and high-performing elderly. Front. Aging Neurosci. 6, 1-10. http://dx.doi.org/10.3389/fnagi.2014.00303.

Vermeij, A., Kessels, R.P.C., Heskamp, L., Simons, E.M.F., Dautzenberg, P.L.J., Claassen, J.A.H.R., 2016. Prefrontal activation may predict working-memory training gain in normal aging and mild cognitive impairment. Brain Imaging Behav., 1-14. http:// dx.doi.org/10.1007/s11682-016-9508-7.

Wang, Y., Zhou, Y., Ma, Y., Leventhal, A.G., 2005. Degradation of signal timing in cortical areas V1 and V2 of senescent monkeys. Cereb. Cortex 15, 403-408. http:// dx.doi.org/10.1093/cercor/bhh143.

Ward, L.M.K., Aitchison, R.T., Tawse, M., Simmers, A.J., Shahani, U., 2015. Reduced haemodynamic response in the ageing visual cortex measured by absolute fNIRS. PLoS One 10. http://dx.doi.org/10.1371/journal.pone.0125012.

Wijeakumar, S., Shahani, U., Simpson, W. a., McCulloch, D.L., 2012. Localization of hemodynamic responses to simple visual stimulation: an fNIRS study. Investig. Ophthalmol. Vis. Sci. 53, 2266-2273. http://dx.doi.org/10.1167/iovs.11-8680.

Wijeakumar, S., Spencer, J.P., Bohache, K.P., Boas, D.A., Magnotta, V.A., 2015 Validating a new methodology for optical probe design and image registration in fNIRS studies. Neuroimage 106, 86-100.

Wijeakumar, S., Huppert, T., Magnotta, V., Buss, A.T., Spencer, J.P., 2017. Validating an image-based fNIRS approach with fMRI and a working memory task. Neuroimage 147, 204-218. http://dx.doi.org/10.1016/j.neuroimage.2016.12.007.

Xu, Y., Chun, M.M., 2006. Dissociable neural mechanisms supporting visual short-term memory for objects. Nature 440, 91-95. http://dx.doi.org/10.1038/nature04262.

Yücel, M. a., Selb, J., Cooper, R.J., Boas, D. a., 2014. Targeted principle component analysis: a new motion artifact correction approach for near-infrared spectroscopy. J. Innov. Opt. Health Sci. 7, 1-8. http://dx.doi.org/10.1142/S1793545813500661.

Zanto, T.P., Rubens, M.T., Thangavel, A., Gazzaley, A., 2011. Causal role of the prefrontal cortex in top-down modulation of visual processing and working memory. Nat. Neurosci. 14, 656-661. http://dx.doi.org/10.1038/nn.2773. 